\title{
Malignancies of the female genital tract from general gynecological services: Five years review at BPKIHS
}

\author{
Hanoon P Pokharel, Nibedita Basnet, Dhruba Uprety, Basudeb Banerjee, \\ Aravind Sinha*, Paras K Pokharel** \\ Department of Obstetrics \& Gynecology, Pathology*, Community Medicine** \\ BP Koirala Institute of Health Sciences, Dharan, Nepal.
}

\begin{abstract}
Aim: To observe the trend of malignant diseases of the female genital tract, and to assess pattern amongst the diagnosed cases and its management at BPKIHS.

Methods: All the diagnosed cases of female genital malignancies were followed. Cases in advance stages were referred to Oncology Centers. Some patients opted to undergo treatment at BPKIHS. Those case sheets and histopathology reports were analyzed to see the trend and pattern of diseases and its optimum management. Referred cases who came for further follow up in the general gynecology department after consultation and treatment are also included in the study.

Setting: Department of obstetrics \& Gynecology and Pathology, BPKIHS.

Results: Cervical cancer was the commonest malignant disease $216(67.3 \%)$ followed by the ovary (17\%), endometrium (10\%), choriocarcinoma (3.4\%) and vulva and vagina (2.8\%). The mean (years in) age of the patients varied with the type of cancer as follows - cervix (30.9), ovary (40.4), endometrium (54.8), choriocarcinoma (25.6) and Vagina \& vulva (42.2).

Out of the total 216 patients Carcinoma Cervix only 20 patients were admitted for Radical surgery and treatment. 196 patients belonged to the group of advanced carcinoma cervix and were referred to the cancer hospital for radiotherapy and chemotherapy.

Conclusion: Cancer cervix is the most common and manageable disease if diagnosed early provided mandatory selective screening program are implemented. The missed opportunity to diagnose the disease in time should be utilized by creating national or regional community - based cervical cancer screening program. General gynecological service provider can also contribute a lot to reduce the burden of disease.
\end{abstract}

Keywords: Female genital malignancies, carcinoma cervix, radiotherapy and chemotherapy.

\section{Introduction}

Endometrial, ovarian and cervical cancers are among the leading causes of death in women.

Malignancies of the female genital tract are increasing in Nepal. ${ }^{1}$ This may be due to increase in awareness and greater numbers of women attending hospital for some or other gynecological problem. The impact of improved screening for $\mathrm{Ca} \mathrm{Cx}$ and $\mathrm{Ca}$ ovary is possibly also a factor.
In Nepal, a country of approximately 23 million people, cervical cancer is more common than breast cancer, and accounts for almost one third of all cancer in females, with more than 2100 new cases being detected each year, (an incidence rate of over 18 per 100,000 per annum). ${ }^{1}$ Unfortunately, most cases present in hospital at a very advanced stage, when treatment is more costly, complicated, and much less likely to be successful. ${ }^{2,3}$ Cervical cancer can easily be detected by several methods - direct visualization of the cervix, direct visualization with application of acetic acid or Lugol's 
iodine to the cervix, or by cytology. Early detection, followed by appropriate therapy could make a very significant difference to survival rates of women with cervical cancer, and would be much more cost effective than focusing on treatment in late stage disease. ${ }^{4}$

There is now consistent evidence that Human Papillomavirus (HPV) has a causal role in the etiology of cervical cancer and those sexual habits and reproductive/hormonal factors are associated with the risk of invasive cervical cancer. ${ }^{5}$ Less clear is the role of dietary habits, but recent studies have shown a direct relationship between smoking and the risk of invasive cervical cancer and an inverse one between selected vitamin intake and risk of the disease. ${ }^{6}$

With reference to ovarian cancer, oral contraceptives and parity, late menopause and lifelong regular menstrual periods increase the risk of the disease. In terms of biologic mechanisms, these factors are thought to act on ovarian cancer risk by affecting lifetime number of ovulations. Along this line, 'incessant ovulation' could be the relevant exposure that defines the incidence of the neoplasm. Among the factors other than reproductive or hormonal, diet is likely to be the most relevant, but it is still however, poorly quantified. ${ }^{6}$

The role of unopposed estrogens in endometrial carcinogenesis is well established. ${ }^{8,9}$ Any factor that increases the exposure of the endometrium to unopposed estrogen, such as menopausal replacement treatment, obesity, or irregular menstrual cycles tends to raise the risk of the disease, while factors that lower exposure to estrogens or increase progesterone levels, such as oral contraceptives (OC) decrease the risk. $^{10-14}$

These well-established risk factors can have different effects at different ages, particularly in preand postmenopausal women: more generally, the interaction between various factors is still not totally defined.

With this background we have done small attempt to see and analyze the current situations of Female Genital Malignancies taken care and referred from our set up, though it does not have any special unit organized, equipped, formally trained personnel for the same.

\section{Objectives}

To observe the trend in malignant diseases of the female genital tract diagnosed cases at BPKIHS and to review the possible management and outcome of those cases. Concept and discussion in the possible role of teaching institution in helping early diagnosis and care.

\section{Methods}

This was a hospital based retrospective study in the departments of Obstetrics and Gynecology and Pathology, BPKIHS. Case sheets and histopathology reports were analyzed to see the trend and pattern of disease and its management. Cases in advanced stage were referred to oncology centres. Almost all patients who were diagnosed as ca ovary and ca endometrium underwent treatment at BPKIHS in the form of Debulking surgery and lymphadenectomy and total abdominal hysterectomy plus bilateral salpingoophorectomy and infracolic omectectomy in case of $\mathrm{Ca}$ Endometrium. Patients who required chemotheraphy or radiotheraphy were referred to Oncology centres. Some patients opted to undergo chemotheraphy in Department of Obs/Gyn.

\section{Results}

Cervical cancer was the commonest site of malignant disease $(67.3 \%)$ followed by the ovary $(17 \%)$, endometrium (10\%), choriocarcinoma (3.4\%) and vulva $\&$ vagina $(2.8 \%)$ (Fig 1). Among the patients of Choriocarcinoma two patients were diagnosed to have choriocarcinoma following delivery of full term healthy babies. They both presented with continuous abnormal bleeding following delivery. All cases of Ca Ovary, Ca Endometrial and Vulva \&Vagina underwent complete surgery in our hospital.

The mean age of the patients varied with the type of cancer as follows - cervix (30.9 years), ovary (40.4 years), endometrium (54.8 years), choriocarcinoma (25.6 years) and Vagina \& vulva (42.2 years).

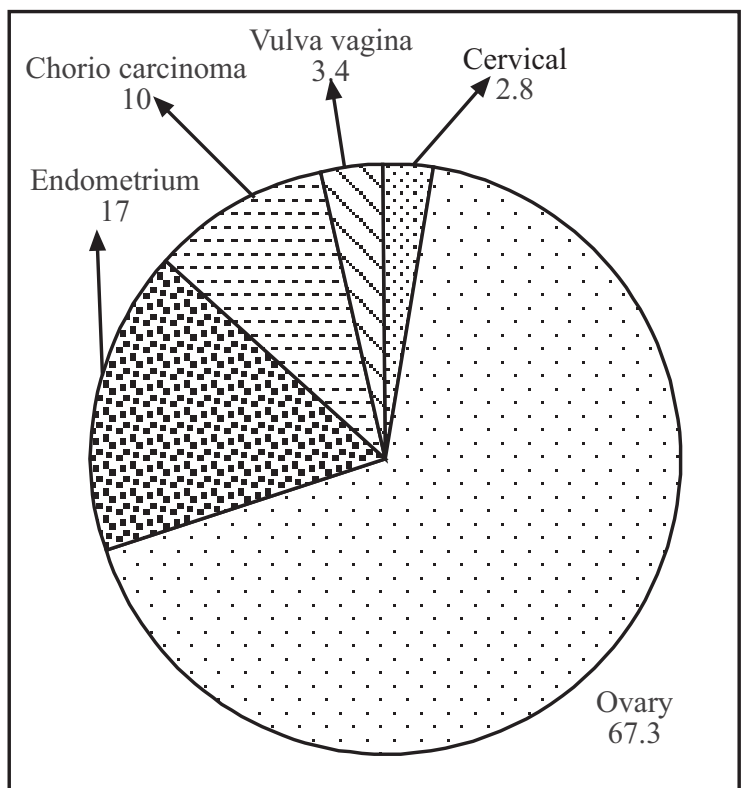

Figure 1. Graphical Presentation of Proportions of malignancy. 
Out of the total 216 patients $\mathrm{Ca} \mathrm{Cx}$, only 20 patients were admitted for Radical surgery and treatment. 196 patients belong to the group of advanced carcinoma cervix and were referred to the cancer hospital for radiotherapy and chemotherapy. At the time of writing, 31 Wertheim's hysterectomies were performed in BP Koirala Institute of Health Sciences.

\section{Discussion}

Cancer of the cervix is the most common and manageable disease if diagnosed early. A general gynecological service provider can contribute to reduce the burden of disease.

Screening for cervical cancer in the non industrialized world is a real challenge, because of the paucity of resources, health awareness amongst the women and inadequate treatment of Cervical Intraepithelial Neoplasia (CIN). Colposcopy and other means of tissue diagnosis like liquid based cytology and HPV testing is not available in many of the centres. However simple technique like visual inspection of the cervix after acetic acid (VIA) may prove helpful in reducing the incidence and mortality from cancer cervix in non industrialized world. ${ }^{5}$

The missed opportunity to diagnose the disease in time often proves fatal. This has huge impacts on family life as well as having a high economical and social cost. A Teaching hospital like ours, can initiate the development of a national or regional community-based cervical cancer screening program integration with primary health care and district health services.

Due to lack of a proper screening programme in the district and zonal hospitals many cases are not diagnosed in time. The Burden of advanced cases of $\mathrm{Ca} \mathrm{Cx}$ is increasing. In our service a small proportion are treated. For the most part, patients need to be referred to a district centre at great inconveniences to them. In case of ovarian cancers most of the patients are stage 3 at the time of presentation. Patients generally attend at BPKIHS for chemotherapy subsequent to surgery here, but many of them are referred to cancer centre due to lack of cancer care unit, especially for radiotherapy.

The sensitivity and specificity of available screening tests for ovarian cancer in asymptomatic women are uncertain and require further study. Although various tests can detect occasional asymptomatic tumors, there is currently no evidence that routine screening will improve overall health outcomes. The large majority of women with abnormal screening test results does not have cancer, yet will require invasive procedures (laparoscopy or laparotomy) to rule out malignancy. Given the risks, inconvenience, and substantial costs of follow-up testing, and the current lack of evidence that screening reduces morbidity or mortality from ovarian cancer, routine screening cannot be recommended. ${ }^{7}$ Trials to determine the benefits and risks of ovarian cancer screening are under way. There is also no evidence to support routine screening in women with a history of ovarian cancer in a first-degree relative. Although such women are at increased risk and stand to benefit more from interventions that reduce ovarian cancer mortality, the effectiveness of screening has yet to be determined for any group of women. Referral to a specialist may be appropriate for women whose family history suggests hereditary ovarian cancer syndrome, due to the very high risk of cancer in this disorder. ${ }^{14}$

The average age for Ovarian cancer to occur is in the $5^{\text {th }}$ decade of life. ${ }^{7,14}$ But in our study we found that the mean age of presentation with ovarian cancer was 40.4 years of age, which is one decade earlier than reported. We suggest that a detailed study in much larger sample and prospective follow up is required. Demographic and cultural factors in the Nepalese population including reproductive patterns, lifestyle, food habits, contraceptive use, genetic predisposition and racial differences to explore the incidence and frequency of neoplasm in early age onset.

In the small numbers of patients who present with nongynecologic symptoms there is a rapid awareness of the possibility of gestational trophoblastic neoplasia; nevertheless, the outcome may be fatal, especially in the presence of symptomatic brain metastases. ${ }^{11}$ Postpartum choriocarcinoma presents mainly with vaginal bleeding, and there is often a delay in diagnosis despite being under the care of gynecologists. ${ }^{12}$

Primary Vaginal carcinoma accounts for only $1 \%$ of all genital tract malignancies ${ }^{13,14}$ It usually present at 50 years with most patients in the sixth, seventh decades of life. In our study we found $2.8 \%$ which is slightly high and the mean age at presentation is 42 years.

\section{Conclusion}

Cancer cervix is a disease which is more prevalent in low socioeconomic groups. It is expected that when living standard improves that the burden will shift to cancer breast and Ovarian cancer. A complete Gynecological check-up with speculum and bimanual examination of all women who attend the Gynecological out-patient department would help identify a possible malignant condition, irrespective of any presenting illness. 
There is no limit to the amount of medical care a state is capable of absorbing. Rationing the resources is the only way to tackle priority health need with fairness and equity. Medical colleges with independent teaching hospitals can also contribute to reduce the public health burden. They can act as catalyst by strengthening weak health system through its rich expertise in human resource.

The dearth of basic resources in Nepal is critical. Most of the district hospital are not equipped with Pathology Unit .Pathology services at Zonal Hospitals are very underdeveloped and many of he histopathological reports are inconclusive.

A collaborative approach between Government and Private Medical Colleges to support local health services would dramatically improve the range and quality of Public Health services. The benefits of sharing expertise and learning between institutions would provide a Win-Win situation for healthcare improvements for the Nepalese population and for medical education advancement.

\section{References}

1. Parkin DM, Bray F, ferlay J et al. Estimating the world cancer burden: Globocan 2000. Int J Cancer 2001;94:153-6.

2. Parkin DM, Muir CS, Whelan SL et al. Cancer incidence in five continents. Vol VI.Lyon: IARC Scientific Publication No 120, 1992.

3. Takeda T ,Sagae S, Koizumi M et al. Multiple primary malignancies in patients with gynecologic cancer. Int J Gynecol Cancer.1995 Jan 5(1); 34-39.

4. Mc Cord ML, Stovall TG, Merrick J L et al.Cervical cytology: A randomized comparison of four sampling methods. Am J Obstet Gynecol 1992;166:1172-9.
5. Ganesan $\mathrm{R}$ and Rollason $\mathrm{T}$. Concepts in gynecology pathology: recent advances and their Clinical relevance. Eur J Surg Oncol.2006 Sep; 32(7):698-706.

6. Parazzini F, Franceshi S, La Vecchia $\mathrm{C}$ et al.The epidemiology of ovarian cancer.Gynecol Oncol 1991; 43:9-23.

7. Risch HA,Weiss NH,Lyon JL et al.Events of reproductive life and the incidence of epithelial ovarian cancer.Am J.epidemol. 1983; 117: 128-39.

8. Enriori CL, Reforzo-membruis J. Peripheral aromatization as a risk for breast and endometrial Cancer in postmenopausal women: a review. Gynaecol oncol 1984; 17:1-21.

9. Lyon JL, Gardner JW. The rising frequency of hysterectomy:its effect on Uterine Cancer rates.Am J epidem. 1977; 105:439-43.

10. Jordan VC. Tamoxifen and Tumorigenicity. A predictable concern.J Natl cancer. Inst 1995; 87:623-9.

11. Baker, Vicki V. "Gestational Trophoblastic Disease.” In Clinical Oncology, 2nd ed. Ed. Martin D. Abeloff, et al. Philadelphia: Churchhill Livingstone, 2000.

12. Nugent D,Hassadia AEverard J et al.Postpartum choriocarcinoma presentation, management and survival.J Reprod Med.2006 Oct;51(10):819-24.

13. Ikenberg H,Runge M,Goppinager A et al.A Human Papillomavirus DNA in Invasive Carcinoma of the Vagina.Obstet Gynecol 1990;432-437.

14. Jonathan S B,Eli YA, Paula AH.Novak's Gynaecology.12 $2^{\text {th }}$ Edition.1057-1261. 